\title{
Apprendre le pan, apprendre à être à Trinidad et Tobago
}

De l'accomplissement personnel à la construction nationale

Learning pan: Learning to be in Trinidad and Tobago. From a personal accomplishment to nation-building

Aprender el pan, aprender a ser. De la realización personal a la construcción nacional en Trinidad y Tobago

\section{Aurélie Helmlinger}

\section{OpenEdition}

\section{Journals}

Édition électronique

URL : https://journals.openedition.org/ries/5959

DOI : 10.4000/ries.5959

ISSN : 2261-4265

\section{Éditeur}

France Education international

Édition imprimée

Date de publication : 1 septembre 2017

Pagination : 87-96

ISBN : 978-2-85420-615-9

ISSN : $1254-4590$

Référence électronique

Aurélie Helmlinger, « Apprendre le pan, apprendre à être à Trinidad et Tobago », Revue internationale d'éducation de Sèvres [En ligne], 75 | septembre 2017, mis en ligne le 01 septembre 2019, consulté le 25 juin 2021. URL : http://journals.openedition.org/ries/5959 ; DOI : https://doi.org/10.4000/ries.5959 


\title{
Apprendre le pan, apprendre à être à Trinidad et Tobago
}

\author{
De l'accomplissement personnel à la construction nationale
}

\author{
Aurélie Helmlinger \\ CNRS
}

Qu'apprend on lorsque on s'initie à la pratique du pan ? Parfois également appelé "steelpan " ou "steeldrum ", cet instrument fut inventé à Trinidad et Tobago pendant la Seconde Guerre mondiale (Stuempfle, 1995 ; Dudley, 2007). Fabriqués à partir de bidons de pétrole recyclés en idiophones mélodiques et accordés sur l'échelle tempérée - comme un piano -, les pans constituent une famille percussive se déclinant du grave à l'aigu dans des orchestres appelés steelbands, aux côtés d'instruments de percussions non mélodiques (batterie, congas, idiophones frappés ou raclés). Comme toute pratique musicale, le jeu en steelband requiert une variété de compétences dans lesquelles le social, le musical et le cognitif (émotions, mémoire, motricité...) s'imbriquent étroitement. Les connaissances musicales stricto-sensu ne sont qu'une facette des domaines d'apprentissage développés par la pratique de la musique. Une vue d'ensemble de ce champ de compétence nécessite par conséquent une approche pluridisciplinaire. Si le présent travail s'inscrit avant tout dans le champ de l'ethnomusicologie, il puise ses ressources théoriques parmi des disciplines variées, allant de l'anthropologie à la psychologie cognitive de la musique. On tentera surtout de tirer les fils qui relient ces éléments.

Dans un premier temps, c'est à la transmission musicale en elle-même - et à ce qu'elle implique cognitivement - que l'on s'intéressera ; en second lieu, on analysera ce que l'organisation des orchestres - et de la façon dont ils répètent - développe comme compétence sociales, notamment en termes de discipline et de patience; enfin, on montrera comment l'inscription des steelbands dans des réseaux nationaux fait de la pratique du pan un agent politique qui non seulement a une place privilégiée dans le roman national, mais a permis un véritable transfert de rapports violents vers la pratique musicale.

\section{APPRENDRE UN MORCEAU : "SHARE THE MUSIC »}

À Trinidad et Tobago, les personnes souhaitant jouer du pan passent forcément - à de rares exceptions près - par le steelband, donc par un collectif. En effet, les instruments sont très rarement possédés par les musiciens, et qui veut apprendre doit se rendre dans un panyard, le lieu de répétition du steelband. 
C'est alors généralement "l'arrangeur» (le directeur musical) ou le captain (responsable du groupe) qui dirige les répétitions, et donc qui est chargé de la transmission. Ces groupes, qui jouent le plus souvent dans des compétitions nationales, en particulier celle du Panorama ${ }^{1}$, jalonnent le territoire trinidadien. Ils sont le lieu privilégié de l'apprentissage, à moins que le débutant ne s'initie - même si c'est plus rare - dans une église, ou maintenant, de plus en plus souvent, en milieu scolaire. En effet, depuis 2004, sous l'impulsion du gouvernement, un programme appelé "Pan in the Classroom» a permis la création de steelbands dans des écoles, dirigés par des enseignants de pan; seule une minorité d'établissement bénéficie encore de ce programme. Si les environnements religieux ou scolaires diffèrent sur bien des aspects de celui des panyards, les modes de transmission ont en réalité beaucoup en commun. En particulier, ils partagent tous la caractéristique de placer d'emblée le paniste - musicien de pan - dans un ensemble orchestral.

Que ce soit au panyard, à l'église ou à l'école, la part formelle de l'enseignement se résume en effet essentiellement à l'apprentissage du répertoire. Cette règle connaît toutefois une exception : l'échelle chromatique, qui peut être soit explicitement transmise, soit connue du fait que, comme elle est souvent utilisée dans la musique (Helmlinger, 2001), les panistes savent généralement à quoi ce terme se réfère. Ils apprennent donc, en principe, comment monter du grave à l'aigu sur leur instrument. Dans les écoles, certains enseignants s'attachent maintenant aussi à faire connaître des gammes diatoniques, mais cette part un peu plus théorique de la transmission est en général rapide et loin d'être systématique.

Pour ce qui est des apprentissages moteurs, c'est au fil de l'acquisition $\mathrm{du}$ répertoire et sans guère d'encadrement verbal que le paniste acquiert la souplesse du jeu de poignet nécessaire à la manipulation de mailloches dans un espace concave. Si un principe général peut être énoncé (jouer les notes placées à droite de la main droite, et les notes de gauche de la main gauche), le novice est en fait laissé très libre dans ses doigtés. Ses choix sont cependant influencés par l'observation des gestes de panistes expérimentés qu'un enfant trinidadien peut faire depuis l'enfance : la taille de l'instrument, qui est composé de un à douze bidons pour un musicien, rend les stratégies motrices particulièrement visibles. Si le débutant n'a pas encore la musculature adéquate, sa posture et ses choix de mouvements montrent qu'une part de transmission des savoir-faire moteurs est déjà effectuée en amont, ce qui tend à confirmer qu'une grande partie de l'apprentissage a lieu hors des situations d'enseignement formelles (Atran et Sperber, 1991). La norme technique est essentiellement déterminée par l'efficacité du geste, sa capacité à être effectué au tempo, et seules les aberrations

1. Compétition nationale associées aux festivités du carnaval. Il s’agit du principal événement musical des steelbands de Trinidad et Tobago. Rassemblant de 150 à 200 groupes selon les années, le nombre de musiciens oscille entre une trentaine à une centaine par steelband. 
seront relevées et corrigées : si le musicien frappe vraiment trop fort, faisant saturer la note de son instrument ${ }^{2}$ («don't slam the pan!»), risquant ainsi de le désaccorder, ou s'il est bloqué par un doigté infaisable à la vitesse requise, encore que le novice n'obtienne pas forcément une aide explicite dans ce cas de figure. Lorsqu'une partie musicale nécessite une musculature qu'il n'a pas encore développée, on lui suggère parfois de se muscler, hors instrument, avec des baguettes de métal. C'est à peu près tout, pour ce qui concerne les savoirs moteurs, qui seront acquis au fil de la pratique. L'immense majorité des répétitions est consacrée à la mémorisation des pièces.

Pour la transmission, la part d'échanges inter-individuels avec un transmetteur est réduite au strict minimum. L’orchestre étant divisé en "sections ", ensemble d'instruments identiques et jouant à l'unisson, c'est à ce sous-groupe que le transmetteur s'adresse généralement, ou à son responsable, le "section leader", qui doit ensuite enseigner à ses camarades ce qu'il a appris. Outre ces séances collectives, des transmissions inter-individuelles sont très souvent nécessaires. Pour la compétition du Panorama notamment, de nombreux panistes arrivent au fur et à mesure des répétitions, et doivent alors rattrapper le morceau avec un paniste de leur section. Comme je l'ai montré ailleurs, la démonstration visuelle est essentielle à la transmission : les surfaces accordées sur l'instrument ayant une forme et une taille propres, elles ont chacune leur identité visuelle (Helmlinger, 2012). Le son est bien sûr crucial, comme toujours en musique, mais il ne suffit ici pas à enseigner, et l'aide visuelle est particulièrement efficace : les musiciens regardent la succession de gestes pour pouvoir l'imiter, et cela jusqu'à un bon niveau de pratique. Ce renfort visuel facilite probablement la mémorisation puisque ce paramètre sensoriel est connu en sciences cognitives pour sa robustesse dans ce domaine (Eysenck, 2012 ; Squire et Kandel, 2002). Certains panistes, notamment les improvisateurs, finissent bien sûr par pouvoir jouer d'oreille, mais il s'agit d'une habileté rare et admirée.

Sans base théorique, c'est en effet bien une sorte de chorégraphie, que le musicien doit retenir, lorsqu'il apprend à jouer une pièce, guidé dans son apprentissage par les gestes du transmetteur ou de ses camarades. La mémoire semble particulièrement efficace puisque les musiciens n'ont ni la possibilité de varier - comme cela peut être le cas pour les formes les plus complexes dans les traditions orales - ni l'aide de la partition (Helmlinger, 2012). Ils jouent rigoureusement par cœur. Il leur faut aussi jouer sur des instruments dont la logique sous-jacente est assez contre-intuitive, puisque contrairement à beaucoup d'instruments, les notes ne sont pas disposées linéairement du grave à l'aigu. Les contraintes d'accordage ont poussé les fabricants à éloigner les intervalles jugés "dissonants", en particulier les secondes mineures et majeures, donc des petits intervalles, et à placer au contraire de manière adjacente préférentiellement des intervalles plus grands tels que des quintes, quartes, ou tierces,

2. Lors d'une frappe très forte, la hauteur de la note est altérée. 
les octaves étant maintenant systématiquement rassemblées. Les repères musicaux sont donc délicats à assimiler pour les panistes, ce qui contribue probablement à expliquer la difficulté qu'ils semblent éprouver à jouer d'oreille. Le paniste se sert donc beaucoup de son sens visuel. Avec l'expérience, toutefois, même lorsqu'il ne pousse pas, comme les plus passionnés, la curiosité jusqu'à s'instruire en théorie musicale, le paniste développe une certaine connaissance de son instrument. Sans acquérir nécessairement la capacité à jouer d'oreille, qui implique une connaissance, même non verbale, des intervalles, les processus de mémorisation semblent de plus en plus courts. Tout se passe comme si les expériences passées lui permettaient de reconnaître des figures musicales ou intervalles et d'accélérer la mémorisation, mais qu'il ne s'agissait pas d'une connaissance suffisante pour déduire une séquence motrice d'une audition musicale (jeu d'oreille). On peut supposer que le paniste acquiert au fil du temps de ce que les sciences cognitives appellent une mémoire implicite de son instrument (Eysenck, 2012 ; Squire et Kandel, 2002), facilitant la mémoire mais insuffisante pour le jeu d'oreille.

Dès que les panistes ont retenu la succession de notes - c'est-à-dire en quelques minutes -, la partie sera répétée en boucle avec les camarades de section ou même directement avec le reste de l'orchestre. L'une des qualités essentielles qu'ils devront développer pour cela est le sens rythmique, qui leur permettra d'honorer l'immense exigence de précision culturellement attendue dans la synchronie des frappes. Il faut répéter inlassablement les parties pour atteindre la justesse temporelle demandée et maîtriser les imbrications rythmiques complexes caractéristiques des musiques polyrythmiques : une écoute d'une grande acuité, particulièrement tournée vers les autres, s’impose. Il faut souligner qu'un steelband peut comprendre jusqu'à une centaine de musiciens, et que s'ils peuvent jouer avec un chef d'orchestre, celui-ci accompagne la musique sans réellement la diriger. L'immense précision de la coordination temporelle de l'ensemble repose essentiellement sur les capacités rythmiques culturellement développées à Trinidad et Tobago, conjuguée aux nombreuses heures de pratique pendant lesquelles le musicien adapte, par les mouvements de son corps, la logique polyrythmique à l'espace de jeu en trois dimensions des pans. Enfin, l'un des aspects les plus remarquables des performances mnésiques des panistes est qu'elles sont étroitement dépendantes du collectif. J'ai en effet pu observer les différences radicales de qualité des performances mnésiques entre des situations collectives et d'autres, où le musicien jouait seul. Une expérience inspiré de la psychologie cognitive a confirmé les observations : le jeu en groupe favorise clairement la mémoire, pour les experts comme pour les non experts (Helmlinger, $2010 ; 2012)$.

On voit différentes compétences se dessiner dans l'apprentissage musical en steelband. Il est intéressant de noter que toutes ces compétences s'élaborent étroitement en interaction avec les autres musiciens, elles sont construites avec et par la relation à autrui. 


\section{Discipline et PATIENCE}

Les steelbands sont apparus dans des environnements difficiles, des zones urbaines défavorisées, parmi des populations stigmatisées, pour la plupart des descendants d'esclaves africains ${ }^{3}$, artisans ou ouvriers. Imbriqués dans un système de compétitions musicales organisées à l'échelle du pays, le paniste s'insère, on l'a vu, dans un collectif. Au-delà des aspects strictement liés à l'apprentissage de l'exécution musicale, l'appartenance à cet ensemble comporte plusieurs corollaires en terme d'organisation, qui ont un impact direct sur le comportement du musicien. D’abord, un steelband implique forcément une logistique développée. Les orchestres atteignent une centaine de musiciens. Un seul instrument peut comporter jusqu'à douze bidons entiers, en plus du "rack», la structure métallique qui les assemble. Il faut alors une quinzaine de personnes pour le soulever, un camion pour le transporter. Être membre d'un steelband, c'est donc nécessairement prendre sa place dans une organisation complexe, un réseau d'entraide. Par la force des choses, c'est une école de sociabilité.

Pour l'apprentissage musical également, le musicien doit, pour participer, établir des rapports sociaux : on doit « lui donner» la musique, pour reprendre les termes employés. Lorsqu'il s'agit d'un enseignant missionné par le programme "Pan in the Classroom ", lorsque le transmetteur est l'arrangeur et qu'il est donc particulièrement motivé à ce que tout le monde apprenne efficacement pour pouvoir obtenir la meilleure interprétation possible, ou encore lorsque le transmetteur a vraiment la fibre enseignante, l'opération se déroule en principe de façon optimale. Mais il arrive fréquemment que la transmission à l'intérieur de l'orchestre ne soit pas si simple (Helmlinger, 2011), les transmetteurs se retrouvant en position d'enseignement de façon parfois quelque peu subie. Il est fréquent qu'ils s'ingénient à se faire prier et rendent le processus de transmission plus complexe qu'il pourrait l'être. Retards, rendez-vous manqués, jeu d'esquive, démonstration trop brève... L'apprenti en steelband doit fréquemment développer des trésors de négociation, de patience. Il doit ne pas compter son temps et, le moment venu, faire preuve d'une bonne mémoire. Il doit "gagner » en quelque sorte sa musique, montrer qu'il la mérite par sa soumission aux règles sociales, fussent-elles quelque peu arbitraires. S’il lui est parfois nécessaire de protester de son sort pour obtenir gain de cause, il lui faut toutefois faire bonne figure et maintenir la cohésion de l'orchestre. S'adapter à ce contexte fait partie du parcours initiatique du novice, qui doit ainsi apprendre à apprendre, développer des trésors de diplomatie, de patience voire de fatalisme, et d'efficacité dans l'encodage. Ces qualités sont également requises dans les séances collectives. L'orchestre comprend en effet de quatre à une dizaine de sections instrumentales différentes. Celles-ci rassemblent jusqu'à une ou deux dizaines de musiciens, qui exécutent à l'unisson

3. Selon le dernier recensement (Central Statistical Office, 2011), la composition de la population de Trinidad et Tobago était la suivante : 34,22 \% d'origine africaine, 35,43\% d'origine indienne, $22,8 \%$ de métis, auxquels s'ajoutent 7,55 \% de diverses minorités (caucasiens, chinois, syriens...). 
des parties musicales conjuguant la complexité des polyrythmies d'origine africaine et celle de la musique symphonique occidentale. Il est compréhensible qu'il soit souvent nécessaire de faire jouer parfois longuement certaines sections, sans les autres. Les panistes doivent alors patienter pour une durée indéfinie, tout en restant mobilisables à tout moment, donc sans trop s'éloigner. Parfois, le groupe joue ensemble mais répète inlassablement, des heures durant, un très court passage du morceau. L'ennui monte graduellement, et les musiciens doivent apprendre à surmonter la frustration que cette situation génère inévitablement.

En outre, le contexte des compétitions et les enjeux - prestige et financier - qu'elles portent, conjugué au goût pour la parole dans ces sociétés où l'art du verbe est très valorisé (Abrahams, 1983), génère une abondance de discours parfois longs, destinés à motiver les troupes, à instiller un état d'esprit gagnant. Pour participer à un tel événement, le musicien devra également écouter docilement ces prises de paroles solennelles des responsables, bien qu'ils ne donnent pas toujours d'instructions très concrètes.

L'attente fait à ce point partie des répétitions qu'il est difficile de ne pas y voir une stratégie de susciter la frustration, de la part des responsables, afin d'obtenir non seulement une précision d'exécution touchant à la perfection mais l'investissement émotionnel maximal attendu dans l'interprétation. Les pièces Panorama, notamment, fortement associées au carnaval, se doivent d'être une explosion de joie. Lorsqu'enfin, après les efforts pour apprendre la musique, les heures d'attentes, le moment est venu de jouer, tous ensemble, au tempo voulu, sans interruption, les musiciens laissent exploser une joie de jouer sans mélange, interprètent de tout leur cœur. La formidable intensité de la satisfaction induite par cette réalisation collective justifie enfin tous les efforts investis dans l'apprentissage.

Quoi qu'il en soit, l'expérience du steelband développe nécessairement la capacité à attendre, la discipline, la soumission à un ordre social. Du même coup, elle implique l'apprentissage d'une résistance discrète, sans rébellion. Pour mériter l'exaltant bonheur de jouer dans ce contexte, le musicien doit acquérir un auto-contrôle sans faille. Cette aptitude est un atout essentiel, dans une société où les relations de pouvoir ont conservé une dimension arbitraire probablement héritée du passé esclavagiste du pays. Les rapports sociaux de la région sont marqués par une forte hiérarchisation, et celle-ci s'observe notamment dans l'attente arbitraire qui est imposée aux individus. Ils éprouvent régulièrement le constat de P. Bourdieu (1997) :

Le tout-puissant est celui qui n'attend pas et qui, au contraire, fait attendre. L'attente est une des manières privilégiées d'éprouver le pouvoir.

L'anthropologue K. Birth, dans son ouvrage spécifiquement dédié à la gestion du temps à Trinidad et Tobago, a finement analysé ces processus (Birth, 1999), montrant que le steelband est loin d'être l'unique lieu où l'on peut vivre cette expérience. Mais ces orchestres offrent une formidable motivation pour développer cette compétence. 


\section{APPRentissage politique}

Rien qu'à l'échelle de l'orchestre, l'organisation matérielle et musicale du steelband pour la préparation des compétitions musicales implique intrinsèquement un jeu politique. L'ampleur de la mobilisation sociale appelle une répartition des rôles et des tâches qui met en jeu l'exercice du pouvoir. Être membre d'un steelband, c'est apprendre à fonctionner à cent, prendre sa place dans ce complexe social, la hiérarchie et le respect des aînés. Si certains rôles-clés sont communs à tous les groupes (arrangeur, captain, section leader...), les modes d'attribution de ces fonctions, ainsi que leur fréquence de rotation, varient beaucoup : autocratie, méritocratie, démocratie... Le paniste apprend rapidement à évoluer et à se positionner dans cette école de vie.

Mais être membre d'un steelband, c'est aussi apprendre à fonctionner à l'échelle du pays. De nombreuses recherches sur l'histoire de ces formations musicales montrent à quel point elles ont été à la fois le produit et l'un des vecteurs de la construction nationale (Stuempfle, 1995 ; Dudley, 2007). Le pan, officiellement consacré "instrument national " par le gouvernement en 1992 (Dudley, 2007), a en effet été un agent politique essentiel, en particulier depuis l'indépendance (1962), les politiciens ayant rapidement perçu son caractère emblématique : invention locale, fabriqué à partir d'un objet de récupération, issu des bas quartiers, apparu en contexte oppressif (harcèlement policier)... La pratique du pan fut donc rapidement investie par un interventionnisme politique très actif. Les premières mesures furent mises en place dès le début des années 1950, afin de pacifier les rapports entre les groupes, dont les rivalités pouvaient provoquer des bagarres sanglantes lors du carnaval. La génération des pionniers était en effet issue d'un milieu très proche de la délinquance et avait une image déplorable dans le pays (Stuempfle, 1995 ; Dudley, 2007). L'une des initiatives les plus importantes fut d'encourager la création d'une association nationale des steelbands, dont Pan Trinbago est l'héritière aujourd'hui.

Cette association est en elle-même un véritable organe de politique nationale des steelbands. Chaque groupe membre participe à l'élection des représentants, régionaux ou nationaux. Entièrement financée par le gouvernement, l'association a pour tâche principale d'organiser les compétitions, et notamment la fameuse compétition du Panorama. Les compétitions musicales créent une arène nationale, dans laquelle les groupes se mesurent chaque année, mettant en jeu une hiérarchie constamment renouvelée, un véritable réseau de sociabilité. Il existe une vie politique parallèle à la vie musicale, avec des campagnes électorales aux enjeux relativement importants : le bureau exécutif - salarié à plein temps en plus de divers avantages en nature - gère un budget qui se compte en millions ${ }^{4}$. Il existe donc des professionnels de la politique des steelbands et le

4. En 2011, il était de 25 millions de $\Pi \$(3,2$ millions $€$ ). En 2016, il était de 30 millions de $\Pi \$$, après être monté jusqu'à 34 millions de $\Pi \$$ (4,4 millions $€$ ) en 2015 (Trinidad Express Newspapers, 2011, 2016). 
musicien, même s'il n'a la plupart du temps pas voix au chapitre, puisque seuls deux membres par groupe ont le droit de vote, n'est en général pas sans opinion sur la gouvernance. Par sa participation musicale, le musicien intègre de fait un modèle réduit de politique nationale, avec les mêmes jeux politiques et les mêmes risques en termes de corruption, d'injustice. Il apprend à explorer l'éventail des possibles qui existent entre la soumission et la résistance : passivité, désapprobation en contexte privé, protestation publique, manifestation et action politique (Loublon, 2017).

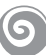

L'intégration dans un steelband développe chez le paniste, une variété de compétences cognitives et sociales; la musique se réalisant - dans sa transmission comme dans toute performance - en une forme de sociabilité. Elle implique à Trinidad et Tobago un investissement souvent discontinu, car lié au carnaval, mais assez chronophage, lorsque le processus d'apprentissage d'un répertoire est en cours. Pour qu'un tel coût soit consenti, le participant doit être attiré par une gratification particulièrement motivante. La transmission culturelle des steelbands semble en effet bénéficier d'une attractivité remarquable, si l'on en croit leur prolifération fulgurante depuis leur création. En un peu plus de sept décennies, ils ont été adoptés par de nombreux pays, et sur tous les continents. Pour reprendre les termes proposé par Olivier Morin (2011), l'attrait d'un phénomène culturel peut être cognitif (facilité de transmission, de reproduction) ou motivationnel (faisant appel à « des émotions, ou des mécanismes de décision, qui nous poussent à la reproduire»). La pratique collective des pans semble offrir un parfait équilibre entre les deux. Les avantages pour la mémorisation facilitent la transmission (Helmlinger, 2012), et l'interprétation très chorégraphique de la musique - en particulier l'heure de gloire de la compétition du Panorama, humblement qualifiée de "greatest show in the world»- suscite l'enthousiasme des participants, et maintient une motivation à toute épreuve.

Ces phénomènes contribuent à expliquer pourquoi les steelbands s'implantent dans des contextes très divers, y compris dans des milieux difficiles, où ils connaissent un succès remarquable. Les exemples abondent : le phénomène semble valable aussi bien à Trinidad et Tobago, où ils ont en premier lieu joué un rôle pacificateur (Stuempfle, 1995 ; Dudley, 2008), qu'aux États-Unis, où ils ont été utilisés par des travailleurs sociaux, dès les années soixante, auprès de jeunes de quartiers comme Brooklyn (Smith, 2012). Plus récemment, ce type d'initiative a été engagé en France. Un steelband a ainsi été initié pour des adolescents évoluant en SEGPA (section d'enseignement général et professionnel adapté) depuis 2010 à La Courneuve ${ }^{5}$, et depuis 2016 à Marigot (Saint Martin) ${ }^{6}$.

5. Alain Rouaud, communication personnelle.

6. Claudie Donne, communication personnelle. 
Dans la région nantaise, une initiative plus ambitieuse a été lancée en 2008, intégrant la pratique du pan dans le cursus scolaire ; actuellement, treize classes de REP (réseau d'éducation prioritaire) de CM1-CM2 sont concernées, soit 254 élèves $^{7}$. Des initiatives ponctuelles ont également lieu en milieu carcéral ${ }^{8}$, avec un succès frappant. À la suite du stage à la prison centrale de Poissy en 2004, les détenus - pourtant condamnés à des peines lourdes et connaissant donc des problèmes de sociabilité particulièrement aigus - ont pris l'initiative de rédiger un document collectif, pour tenter d'obtenir la pérennisation de l'activité : du jamais vu, selon les gardiens, qui avaient pourtant observé les effets d'une grande variété d'activités ${ }^{9}$. Un signe de la formidable adhésion que peut susciter la pratique du steelband et de son rôle socialisant.

\section{BIBLIOGRAPHIE}

ABRAHAMS R. D. (1983): The man-of-words in the West Indies: performance and the emergence of Creole culture, Baltimore, États-Unis/Royaume-Uni.

ATRAN S., SPERBER D. (1991) : Learning without teaching: Its place in culture. In Culture, schooling, and psychological development, p. 3955, Westport, CT, US : Ablex Publishing.

BIRTH K. K. (1999): Any time is Trinidad time: social meanings and temporal consciousness, Gainesville, Fla., États-Unis : University Press of Florida.

BOURDIEU P. (1997), Méditations pascaliennes, Le Seuil.

DUDLEY S. (2007) : "Creativity and Politics in the Steelband Music of Ray Holman, 1957-1972 », in G. L. Green et P. W. : Scher (ed.), Trinidad carnival: the cultural politics of a transnational festival, p. 161-177, Bloomington, États-Unis: Indiana university press.

DUDLEY S. (2008): Music from Behind the Bridge: Steelband Spirit and Politics in Trinidad and Tobago (illustrated edition), New York: Oxford University Press Inc.

EYSENCK M. W. (2012) : Fundamentals of Cognition (2 édition), Hove, East Sussex; New York: Psychology Press.

HELMLINGER A. (2001): "Geste individuel, mémoire collective», Cahiers de musiques traditionnelles, 14, 181-202.

HELMLINGER A. (2010): "Memorizing together. Group effect experiments in steelbands (Trinidad and Tobago)", Annales Fyssen 2: 216-235.

HELMLINGER A. (2011) : "La virtuosité comme arme de guerre psychologique ", Ateliers d'anthropologie, (35).

HELMLINGER A. (2012): Pan Jumbie. Mémoire sociale et musicale dans les steelbands (Trinidad et Tobago), Nanterre : Société d'Ethnologie.

SMITH A. (2012) : Steel Drums and Steelbands: A History, Scarecrow Press.

7. Jean-François Fourichon, directeur de la Maison des Arts de Saint-Herblain, communication personnelle.

8. Différentes initiatives ont été réalisées : à la maison d'arrêt du Val d’Oise en 2003, à la prison centrale de Poissy en 2004, à la maison d'arrêt de la Santé, Paris 2010 (Alain Rouaud, communication personnelle), et également à la maison d'arrêt de Laval en 2015 (Jean Duval, communication personnelle).

9. Alain Rouaud, communication personnelle. 
SQUIRE L., KANDEL E., (2002) : La mémoire: de l'esprit aux molécules, DeBoeck Université, Paris/Bruxelles, $282 \mathrm{p}$.

STUEMPFLE, S. (1995) : The Steelband Movement: The Forging of a National Art in Trinidad and Tobago, Philadelphia : University of Pennsylvania Press.

CENTRAL STATISTICAL OFFICE (2011): Trinidad and Tobago 2011 Demographic Report, p. 402, Trinidad and Tobago: Government of The Republic of Trinidad \& Tobago, Ministry of Planning and Sustainable Development. [https://goo.gl/ovS1qu]

\section{ARTICLES DE PRESSE}

BISHOP V. (07/01/2016) : "Pantrinbago gets $\$ 30$ million », Trinidad Express Newspaper. [https://goo.gl/q8wTuC]

LOUBLON M. (10/01/2017) : "Pan in disharmony as players meet ", Trinidad Express Newspaper. [https://goo.gl/wrjpRa]

Trinidad Express Newspapers (2011, mars 12) : "Pan Trinbago takes lion's share ». [https://goo.gl/dhTH1r] 